\title{
Editorial: Fitness Costs and Benefits of Female Song
}

\author{
Michelle L. Hall ${ }^{1 *}$ and Naomi E. Langmore ${ }^{2}$ \\ ${ }^{1}$ School of BioSciences, The University of Melbourne, Melbourne, VIC, Australia, ${ }^{2}$ Division of Evolution and Ecology, \\ Research School of Biology, Australian National University, Canberra, ACT, Australia
}

Keywords: female birdsong, female fitness, song learning, brain behaviour, female ornaments

\section{Editorial on the Research Topic}

\section{Fitness Costs and Benefits of Female Song}

The complexity and musicality of birdsong has fascinated naturalists for centuries. However, the historical research focus on temperate northern hemisphere birds has led to a widespread perception that song production is the domain of males alone. More recently, studies of birdsong have undergone a major paradigm shift, with the discovery that song in female birds is in fact widespread and ancestral, occurring in over $70 \%$ of surveyed species (Odom et al., 2014). This revolution in our understanding of song evolution has significant implications. First, it casts doubt on the view that song evolved primarily through sexual selection acting on males. Instead, the discovery that song occurred ancestrally in both sexes raises the possibility that song evolved initially for broader, social functions. Second, where sexual dimorphism in song production occurs, it is likely to reflect relatively recent loss of female song from an ancestor that had both male and female song (Odom et al., 2014). This shifts the focus of questions about sexual dimorphism in song production from "why do males sing?" to "why have females of some species lost song?." These issues are addressed in this Research Topic by Price, who argues that recent findings about the prevalence of female song do not conflict with previous research, but instead highlight some problematic assumptions about the evolution of sexual dimorphisms. These include misconceptions that current patterns of elaboration and diversity in each sex reflect past rates of change and that levels of sexual dimorphism necessarily reflect levels of sexual selection.

Given the changing focus in studies of birdsong evolution, a better understanding of how selection acts on song in females is sorely needed. Specifically, we need to understand the fitness costs and benefits of female song, yet there is an almost complete dearth of studies on this topic. The contributions presented in this special issue approach this topic from a diversity of angles, encompassing measures of the relationship between song and fitness, between song and other display traits and between male and female songs, as well as investigations of how females learn their songs.

This article was submitted to Behavioral and Evolutionary Ecology, a section of the journal

Frontiers in Ecology and Evolution

Received: 20 March 2017 Accepted: 29 April 2017

Published: 16 May 2017

Citation:

Hall ML and Langmore NE (2017) Editorial: Fitness Costs and Benefits of Female Song. Front. Ecol. Evol. 5:48.

doi: 10.3389/fevo.2017.00048

\section{FEMALE SONG AND FITNESS}

Two papers in this Research Topic investigate the association between song production and reproductive success in wild birds. Brunton et al. found that in New Zealand bellbirds Anthornis melanura female song rates during both the incubation and chick-rearing phases strongly predicted the number of fledged chicks. Similarly, Cain et al. showed that female song rate in response to playback was positively related to nest success and the number of fledglings produced in superb fairy-wrens Malurus cyaneus. Both these studies provide evidence of a link between female song and fitness. However, further work is needed to identify the nature of this link. Do high song rates and more complex songs improve resource acquisition and increase reproductive success directly, or are 
they independently linked to another variable, such as female condition or age? A third paper sheds some light on this question. Keen et al. demonstrate that in superb starlings Lamprotornis superbus song diversity is correlated with age and breeding experience. In many bird species, nesting success improves with age and there is a concomitant preference for pairing with older mates (Kokko, 1997). This could, in turn, select for song elaboration as a signal of age and experience in both sexes (Langmore et al., 1996; Ballentine, 2009). These studies generate many fascinating hypotheses to explain the relationship between female song, age and breeding success. An important next step is to conduct experimental studies to elucidate the causal nature of the relationship.

\section{HOW DO FEMALES LEARN THEIR SONGS?}

Studying the ontogeny of female song in the wild is challenging because females are usually the dispersive sex in birds (Greenwood, 1980). However, this life-history difference between the sexes may influence the fitness costs and benefits of different song learning strategies if females experience greater changes in social environment associated with natal dispersal. Riebel highlights the importance of studying song learning processes for developing hypotheses for the function and evolution of female song. Knowing who females learn their songs from, and when they learn, are topics explored by three studies in this volume. Evans and Kleindorfer assessed element sharing rates in free-living superb fairy-wrens and found that females, like males, shared elements with both their male and female parents, suggesting that learning in this species does not involve sexspecific lineages. Dalziell and Wellbergen found that female superb lyrebirds Menura novaehollandiae imitate vocalizations of a wide range of other species, and use mimicry in a contextdependent way. Dowling et al. address the question of the timing of sensitive periods for vocal learning, and suggest that vocal learning in red-backed fairy-wrens Malurus melanocephalus may begin very early in life, since offspring songs are similar to maternal incubation calls. With many decades of laboratory studies and increasing numbers of field studies on vocal learning in males, we are just at the beginning of teasing apart song learning in females to discover similarities and differences with what we know about males.

Understanding the biological basis of song in male birds has been vital for investigating the relationship between song and fitness in males. The developmental stress hypothesis linked large song repertoires with large vocal areas in the brain associated with high resource availability during early development, so that repertoire size serves as an honest indicator of male quality (Buchanan et al., 2003). Whether such links between the biological basis of song and fitness exist in female birds is yet to be determined. In this issue, Lobato et al. address the biological basis of song ontogeny in female and male blue-capped cordon-bleus Uraeginthus cyanocephalus with a neuro-anatomical study. Their finding that brain sexual dimorphism is not always paralleled by song dimorphism highlights the importance of considering females as well as males for understanding the complexity of brain-behaviour dynamics.

\section{FEMALE SONG AND OTHER DISPLAY TRAITS}

Another promising avenue for elucidating the fitness benefits of female song is to examine song in the context of other display traits. For example, is female song associated with colourful plumage, or behavioural displays? Webb et al. demonstrate that across songbird species, female song occurs mainly in those species that exhibit elaborate plumage colouration in both sexes. This result suggests that the two signalling modes have reinforcing, overlapping functions and that they have evolved together in response to similar selective pressures. These findings contrast with those of two other studies in this volume. Mahr et al. tested the relationship between song and plumage characteristics in relation to female quality in superb fairy-wrens. They found no relationship between song variables and UV-colouration and proposed that the two traits evolved independently in that species. This may occur when different traits reveal different information about the signaller and are driven by different selective pressures (the "multiple messages" hypothesis). Similarly, Soma and Garamszegi tested the relationship between song, plumage and dance in Estrildid finches and found that the three traits were not correlated in either sex. However, expression of these traits was correlated between the sexes; species that have males with one or more exaggerated traits also have females that express the same traits. Like Mahr et al.'s results, this study suggests that multiple signals arise as a result of different selection pressures.

\section{WHEN FEMALES AND MALES SING}

The effect of communication within and between established breeding partnerships on fitness is often neglected in studies on birdsong. Research on female song sometimes ignores this complexity, either investigating female song function in the same theoretical framework typically used to investigate male song, ignoring the role of the partner, or studying female song in the context of duetting at the level of the pair rather than the individuals. As Logue and Krupp highlight, it is particularly important to distinguish individual- and pair-level perspectives in studies of duetting because if partners combine their vocalisations to form duets, there are multiple pathways by which female song might affect fitness.

Comparative studies identifying life-history and ecological correlates of female song and duetting across a wide range of species can also shed light on the fitness costs and benefits of female song. Previous work has shown that gains of migratory behaviour are typically associated with losses of female song and duetting (Logue and Hall, 2014). However, Najar and Benedict's comparative analysis show that, in a group where migration is the ancestral state, losses of migratory behaviour are not associated with gains of female song. They highlight the need for information about pair bond duration and territoriality throughout the annual cycle to better understand the evolution of female song. Tobias et al. also use phylogenetically controlled comparative analysis to assess evolutionary correlates of duetting and chorusing at a global scale. Including both songbirds and 
non-songbirds, their analysis shows that the likelihood of females vocalising together with males and other group members is most strongly associated with year-round territoriality, and not with latitude, migration, climate variables, or habitat openness.

Thus far, comparative analyses have investigated evolutionary correlates only of the presence or absence of female song. To better understand factors driving the elaboration or loss of acoustic ornaments, we need to know more about variation in female song rates and complexity within and across species. Odom et al. provide such valuable information in a withinspecies comparison of the structure and contexts of female and male song in the Venezuelan troupial Icterus icterus. Studies on female song often involve species with elaborate female song or duets, with less known about cases where female song is not as elaborate. In banded wrens Thryophilus pleurostictus, where females sing at much lower rates and with less complexity than males, Hall et al. find that female song seems to function primarily for communication between partners, rather than to repel rival females or to attract males. However, further work is needed to determine if and how female song used in this context affects fitness.

\section{CONCLUSION}

The diversity of contributions to this Research Topic highlight the breadth of research currently being conducted on female song. They also reveal how much further behind we are

\section{REFERENCES}

Ballentine, B. (2009). The ability to perform physically challenging songs predicts age and size in male swamp sparrows, Melospiza georgiana. Anim. Behav. 77, 973-978. doi: 10.1016/j.anbehav.2008.12.027

Buchanan, K. L., Spencer, K. A., Goldsmith, A. R., and Catchpole, C. K. (2003). Song as an honest signal of past developmental stress in the European starling (Sturnus vulgaris). Proc. R. Soc. B 270, 1149-1156. doi: 10.1098/rspb.2003.2330

Greenwood, P. J. (1980). Mating systems, philopatry and dispersal in birds and mammals. Anim. Behav. 28, 1140-1162. doi: 10.1016/s0003-3472(80)80103-5

Kokko, H. (1997). Evolutionarily stable strategies of age-dependent sexual advertisement. Behav. Ecol. Sociobiol. 41, 99-107. doi: 10.1007/s002650050369

Langmore, N. E., Davies, N. B., Hatchwell, B. J., and Hartley, I. R. (1996). Female song attracts males in the alpine accentor Prunella collaris. Proc. R. Soc. B 263, 141-146.

Logue, D. M., and Hall, M. L. (2014). Migration and the evolution of duetting in songbirds. Proc. R. Soc. B 281:20140103. doi: 10.1098/rspb.2014.0103 in understanding the functions, ontogeny, mechanisms, and evolution (Tinbergen, 1963) of birdsong when it comes to females, compared to males. We hope that this Research Topic stimulates further research to improve our understanding of the fitness costs and benefits of birdsong with more work on females to redress this imbalance.

\section{AUTHOR CONTRIBUTIONS}

$\mathrm{MH}$ conceived the topic idea, and $\mathrm{MH}$ and NL developed the topic, contributed to overseeing peer review of contributions, and wrote the editorial article.

\section{FUNDING}

NL and $\mathrm{MH}$ are funded by the Australian Research Council (DP150101652) and MH is funded by the Australia and Pacific Research Council (APSF14/6).

\section{ACKNOWLEDGMENTS}

We wish to thank all the contributing authors for their efforts, and all those attending symposia on female song in 2015 for their excitement about the topic and stimulating discussions at the Behaviour conference in Australia and meeting of the International BioAcoustics Council (IBAC) in Germany.

Odom, K. J., Hall, M. L., Riebel, K., Omland, K. E., and Langmore, N. E. (2014). Female song is widespread and ancestral in songbirds. Nat. Commun. 5, 3379. doi: $10.1038 /$ ncomms4379

Tinbergen, N. (1963). On aims and methods of ethology. Zeitschrift fur Tierpsychologie 20, 410-433.

Conflict of Interest Statement: The authors declare that the research was conducted in the absence of any commercial or financial relationships that could be construed as a potential conflict of interest.

Copyright (C) 2017 Hall and Langmore. This is an open-access article distributed under the terms of the Creative Commons Attribution License (CC BY). The use, distribution or reproduction in other forums is permitted, provided the original author(s) or licensor are credited and that the original publication in this journal is cited, in accordance with accepted academic practice. No use, distribution or reproduction is permitted which does not comply with these terms. 